\title{
Practical Methods to Assess Chronic Constipation
}

\author{
Kyung Ho Song \\ Division of Gastroenterology and Hepatology, Department of Internal Medicine, Konyang University College of Medicine, Daejeon, Korea
}

\section{Article: Relevance of colonic gas analysis and transit study in patients with chronic constipation Park SY, Park HB, Lee JM, et al}

(J Neurogastroenterol Motil 2015;21:433-439)

Defining the pathophysiology of chronic constipation is critical for planning management. After excluding organic diseases, physician should speculate whether the constipated patient has a defecatory disorder or not. Recently, American Gastroenterology Association suggested an updated algorithm in diagnosis of chronic constipation. ${ }^{1}$ The major change was anorectal manometry and a rectal balloon expulsion test as the initial step which should be considered even before conventional laxative trial especially in patients who are highly suspected as having a defecatory disorder. This change inevitably posed the measurement of colon transit time in the last step of the algorithm and assessment of colonic transit is not recommended as early study any more. What is the rationale of this change? The American Gastroenterology Association medical position statement provided two reasonable issues. First, because near half of patients with defecatory disorders show concomitant slow colonic transit, slow transit time cannot rule out the presence of defecatory disorders and omit anorectal studies. Defecatory disorders are treated with pelvic floor retraining behavioral therapy, namely biofeedback, even if slow colonic transit is concomitant. Second reason is that the administration of laxative is the main initial therapy regardless of colon transit if there is no evidence of defecatory disorders. Normal transit constipation and slow transit constipation are treated similarly.

Then, what is the clinical impact of slow colonic transit? We select very exceptional case of refractory chronic constipation as a candidate of surgical treatment according to the colonic transit. Colectomy could be effective in medically refractory constipated patients with slow colonic transit if defecatory disorders are not concomitant. The overall satisfaction rate of surgery was about $86 \%$ in a systematic review involving 39 reports and 1423 patients. ${ }^{2}$ Confirming slow colonic transit and ruling out defecatory disorders are mandatory for the successful outcome. The present study by Park et $\mathrm{al}^{3}$ suggested that the gas volume scoring could be a practical method assessing colonic transit. Even though the authors cannot find a positive correlation of colon

Received: June 9, 2015 Revised: None Accepted: June 18, 2015

(c) This is an Open Access article distributed under the terms of the Creative Commons Attribution Non-Commercial License (http://creativecommons. org/licenses/by-nc/4.0) which permits unrestricted non-commercial use, distribution, and reproduction in any medium, provided the original work is properly cited.

*Correspondence: Kyung Ho Song, MD Division of Gastroenterology and Hepatology, Department of Internal Medicine, Konyang University Hospital, 158, Gwanjeodong-ro, Seo-Gu, Daejeon 302-718, Korea Tel: +82-42-600-8837, Fax: +82-42-600-9090,E-mail: postit@kyuh.ac.kr, postit2@daum.net

Financial support: None.

Conflicts of interest: None.

ORCID: Kyung Ho Song, http://orcid.org/0000-0001-6661-3879. 
transit time and colon gas volume score, they demonstrated that the difference of gas volume existed between slow transit and normal transit, defined as 45 hours (mean value +1 standard deviation). The difference of gas volume score was small, $5.66 \%$ vs $4.15 \%$, and the sensitivity and specificity of the cut-off value to diagnose slow colon transit was disappointing. The failure of presenting valuable cut-off colon gas volume score to differentiate slow colon transit is a big drawback of this study, because there was only small mean difference of colon gas volume score between the groups and this small difference can be easily made by manual processing of gas volume scoring or by confusing small bowel gas as colon gas. Also the range of colon gas volume score largely overlaps each other. Therefore the present outcome has insufficient clinical impact to replace conventional method using radiopaque markers to assess colon transit time.

But primary physicians can roughly discriminate slow transit constipation based on this tendency of colon gas on simple x-ray and colon transit. When primary physicians encounter a constipated patient with a relatively larger amount of colon gas on simple abdominal x-ray, they can suspect the patient may have slow colon transit. There are some practical methods to assess constipated patient and this include digital rectal exam and Bristol stool scale. We can consider additional anorectal tests in patients of abnormal digital rectal exam. ${ }^{4}$ Abnormal digital rectal exam by skillful examiner can practically suspect defecatory disorder. Also Bristol stool scale is a practical indicator of colon transit and this simple illustration can be useful in a clinical setting of discrepancy between the bowel frequency and stool hardness. ${ }^{5}$ Besides of this physical exam and simple illustration, simple abdominal radiography may give some additional information to primary physician.

\section{References}

1. Bharucha AE, Pemberton JH, Locke GR 3rd. American Gastroenterological Association Technical Review on Constipation. Gastroenterology 2013;144:218-238.

2. Arebi N, Kalli T, Howson W, Clark S, Norton C. Systematic review of abdominal surgery for chronic idiopathic constipation. Colorectal Dis 2011;13:1335-1343.

3. Park SY, Park HB, Lee JM, et al. Relevance of colonic gas analysis and transit study in patients with chronic constipation. J Neurogastroenterol Motil 2015;21:433-439.

4. Tantiphlachiva K, Rao P, Attaluri A, Rao SS. Digital rectal examination is a useful tool for identifying patients with dyssynergia. Clin Gastroenterol Hepatol 2010;8:955-960

5. Saad RJ, Rao SS, Koch KL, et al. Do stool form and frequency correlate with whole-gut and colonic transit? Results from a multicenter study in constipated individuals and healthy controls. Am J Gastroenterol 2010;105:403-411. 